\title{
Histological verification of the usefulness of magnifying endoscopy with narrow-band imaging for horizontal margin diagnosis of differentiated-type early gastric cancers
}

\author{
Makomo Makazu ${ }^{1} \cdot$ Kingo Hirasawa $^{1}$ (D) Chiko Sato $^{1} \cdot$ Ryosuke Ikeda $^{1} \cdot$ \\ Takehide Fukuchi $^{1}$ - Yasuaki Ishii ${ }^{1} \cdot$ Ryosuke Kobayashi $^{1} \cdot$ Hiroaki Kaneko $^{1}$ • \\ Masataka Taguri $^{2} \cdot$ Yoko Tateishi $^{3} \cdot$ Yoshiaki Inayama $^{4} \cdot$ Shin Maeda $^{5}$
}

Received: 15 February 2017/Accepted: 25 May 2017/Published online: 21 June 2017

(C) The International Gastric Cancer Association and The Japanese Gastric Cancer Association 2017

\begin{abstract}
Background Although magnifying endoscopy with narrow-band imaging (ME-NBI) can help identify the horizontal margin (HM) of early gastric cancer (EGC), little is known about the factors that can clarify the HM by using ME-NBI. We aimed to characterize the pathological features of lesions in which the HM was identified using MENBI.

Methods The HMs of 639 differentiated-type EGCs treated with endoscopic submucosal dissection or surgery were analyzed using conventional endoscopy and ME-NBI. The number and width of the intervening parts (IP) and the number, width, and depth of the subepithelial capillaries (SEC) in cancerous and noncancerous areas were measured.

Results In 13 lesions (2.0\%), more than $90 \%$ of the HM was not recognized with conventional endoscopy, but 11 of
\end{abstract}

Kingo Hirasawa

kingo-h@urahp.yokohama-cu.ac.jp

1 Division of Endoscopy, Yokohama City University Medical Center, 4-57 Urafune-cho, Minami-ku, Yokohama 232-0024, Japan

2 Department of Biostatistics, Yokohama City University School of Medicine, 3-9 Fukuura, Kanazawa-ku, Yokohama 236-0004, Japan

3 Department of Pathology, Yokohama City University Graduate School of Medicine, 3-9 Fukuura, Kanazawa-ku, Yokohama 236-0004, Japan

4 Diagnostic Pathology, Yokohama City University Medical Center, 4-57 Urafune-cho, Minami-ku, Yokohama 232-0024, Japan

5 Department of Gastroenterology, Yokohama City University Graduate School of Medicine, 3-9 Fukuura, Kanazawa-ku, Yokohama 236-0004, Japan these lesions were detectable with ME-NBI (NBI group). The HMs of the other 626 lesions were mostly recognized using conventional endoscopy (WLI/CE group). In the NBI group, the IP width, standard deviation (SD), and number of IPs did not significantly differ between the cancerous and noncancerous areas. However, the SEC number was significantly larger and the depth was shallower in cancerous areas. In the WLI/CE group, the IP width and SD were significantly larger, but the IP number was significantly smaller in cancerous areas. The SEC depth was significantly shallower in cancerous areas.

Conclusions Differences of IP width, SD, and IP number may be factors for identifying HMs with conventional endoscopy. Because NBI can better visualize vessel structures, the increased SEC number and shallow SECs may clarify the HM.

Keywords Narrow-band imaging - Early gastric cancer Horizontal margin · Intervening part · Subepithelial capillary

\section{Introduction}

The development of innovative early gastric cancer (EGC) treatments such as endoscopic submucosal dissection (ESD) preceded the end of the twentieth century [1]. ESD is a method widely used to treat EGC with low invasiveness. Diagnosis of the horizontal margin (HM) of superficial gastric cancer is critical to successful treatment. Moreover, precise estimation of the HM can allow for the selection of surgical or endoscopic treatment. Precisely diagnosing the HM can allow surgical treatment to be divided into total and distal gastrectomy. Although conventional diagnostic imaging such as white-light imaging 
(WLI) or chromoendoscopy (CE) has been used, the precise diagnosis of EGCs has been revolutionized by the development of image-enhanced endoscopy [2]. Gastric cancer that has no apparent elevation or depression compared with the surrounding nonneoplastic mucosa is referred to as superficial flat-type cancer (0-IIb) [3]. However, using WLI or CE with indigo carmine dye to recognize HM in superficial, flat-type gastric cancer is often difficult [4]. In such cases, magnifying endoscopy with narrow-band imaging (ME-NBI) is sometimes effective [5]. NBI is an optical technology developed by Olympus that modifies the wavelength and bandwidths of the light of an endoscope light into narrow-band illumination of $415 \pm 30$ and $540 \pm 30 \mathrm{~nm}$ and enhances the microsurface patterns and microvessel patterns of the gastric mucosa. Yao et al. proposed the vessel plus surface (VS) classification that is widely used for the endoscopic diagnosis of EGC. In this classification, if an endoscopist observes an irregular microsurface pattern or an irregular microvascular pattern, or both, together with a clear demarcation line (DL) in a gastric lesion, they can diagnose the lesion as gastric cancer [6]. Clinically, we rarely encounter EGCs in which the horizontal margins are difficult to recognize for almost all circumferences with the use of WLI or CE. In such cases, it was previously necessary to perform many biopsies before treatment to detect the margin histologically. NBI enables the detection of the $\mathrm{HM}$, even in such lesions, and avoids the need for many biopsies. Although the usefulness of the VS classification has been demonstrated in several studies [6-8], the designation of "irregularity" depends on the subjective opinion of each endoscopist. Thus, both inexperienced and experienced endoscopists sometimes have difficulty establishing the DL.

Based on these issues, we wanted to know why NBI enables us to recognize the difference between the cancerous and noncancerous areas in these lesions. However, few reports have evaluated the mechanism by which NBI enables the existence or horizontal extent of such lesions to be recognized. In the present study, we investigated resected specimens of EGCs in which more than $90 \%$ of the HM circumference was undetectable on WLI or CE but could be observed with ME-NBI. We also aimed to characterize the pathological features of the lesions with HMs that were were identified using ME-NBI.

\section{Patients and methods}

\section{Case selection}

Figure 1 is a diagram of the flow of patient recruitment. We treated 558 consecutive EGCs with ESD and 178 with surgical resection between January 2011 and December 2013 at Yokohama City University Medical Center. Among them, differentiated-type EGCs were predominant in both ESD cases (538 lesions) and surgical cases (121 lesions). We defined tubular adenocarcinoma and papillary adenocarcinoma as differentiated-type gastric cancer and poorly differentiated adenocarcinoma, signet-ring cell carcinoma, and mucinous adenocarcinoma as undifferentiated-type gastric cancer according to the Japanese Gastric Cancer Treatment Guidelines [9]. After excluding lesions that did not undergo ME-NBI, we analyzed the HM of 639 lesions. In 13 lesions $(2.0 \%)$, more than $90 \%$ of the circumference of the HM was not recognized with conventional endoscopy. Among those lesions, the HMs of 11 lesions were entirely detectable by ME-NBI (NBI group), whereas the HMs of 2 lesions remained unclear. Of the 2 lesions that were undetectable with both conventional endoscopy and ME-NBI, 1 lesion demonstrated "crawling" differentiated-type gastric cancer, and the other lesion was notably modified by ulcer formation. Of the 11 cases in the NBI group, 5 were treated by ESD and 6 were treated with surgery. In the NBI group, all patients showed predominant macroscopic type 0-IIb disease. Even if the lesion was accompanied by a protruding or indented component, all the lesions of the NBI group were surrounded by type 0 -IIb tissue. As a comparison group, we used 28 size-matched EGCs that were clearly recognized with conventional endoscopy (WLI/CE group). This group included 15 cases of type 0-IIa and 13 cases of type 0-IIc.

All patients were fully informed about the treatment and histopathological examination and provided consent. Our institutional review board approved the study.

\section{Endoscopic examination}

We used the Evis Lucera Spectrum endoscopy system from January 2011 to October 2012 and the Evis Lucera Elite system from November 2012 on (Olympus Medical Systems, Tokyo, Japan), along with high-resolution optical magnifying endoscopes (GIF-H260Z; Olympus Optical, Tokyo, Japan) for NBI. We used a black soft hood attachment (MB46; Olympus) on the tip of the scope in all cases. All patients were initially examined under conscious sedation using intravenous injections of midazolam $(0.05-0.1 \mathrm{mg} / \mathrm{kg})$ to observe the EGC clearly. At the endoscopist's discretion, an additional $2 \mathrm{mg}$ midazolam was given when necessary. After carefully washing the gastric mucosa with water, we observed all locations in the stomach. After finding the target lesion, we observed the macroscopic type, color, and horizontal extent of the lesion using WLI, and then observed the same features of the lesion with CE. We occasionally tried to recognize the HM with a magnified view in WLI 


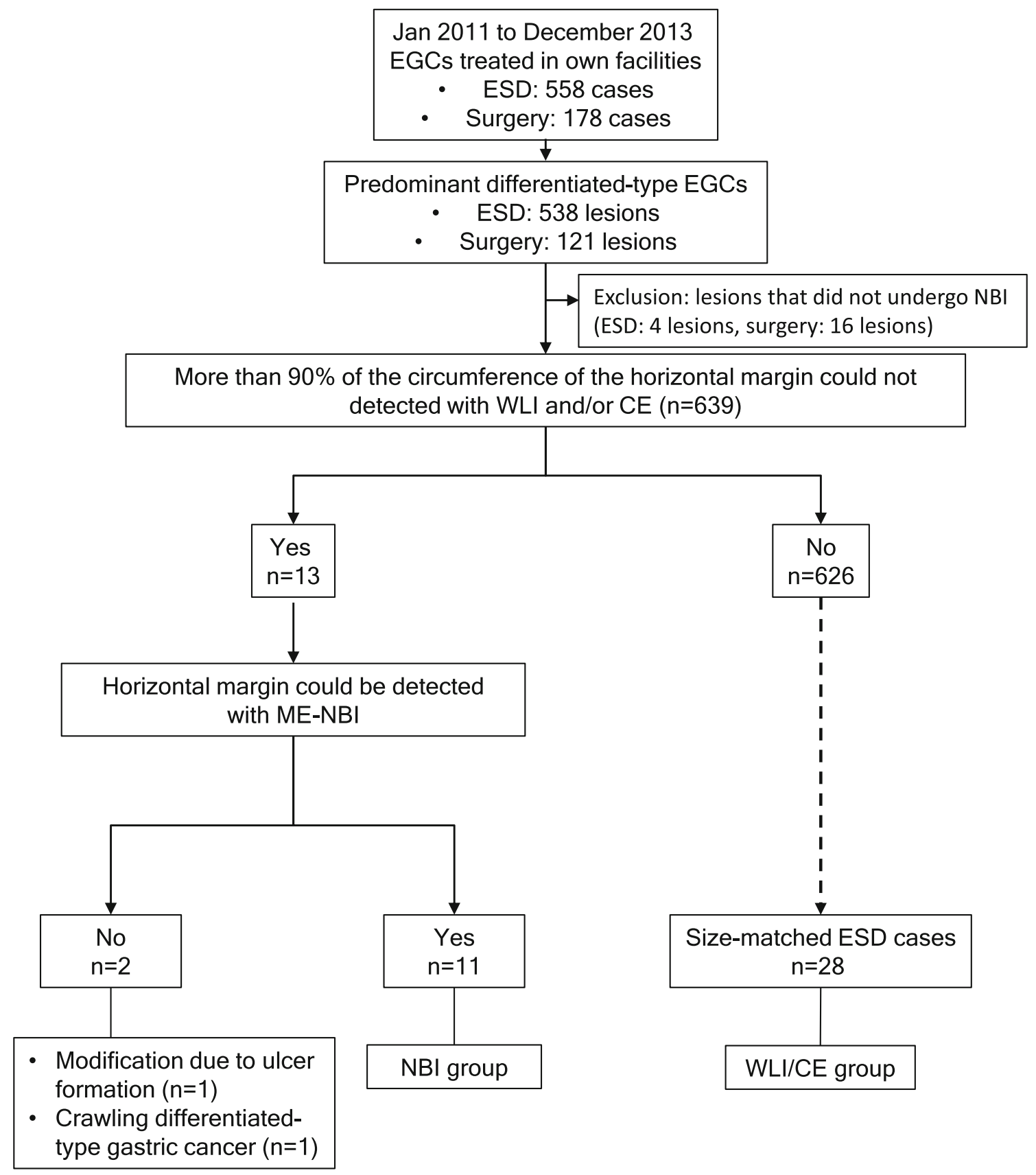

Fig. 1 Patient recruitment. $E G C$ early gastric cancer, $E S D$ endoscopic submucosal dissection, $W L I$ white-light imaging, $C E$ chromoendoscopy, $M E-N B I$ magnifying endoscopy with narrow-band imaging

and CE. Then we observed lesions with ME-NBI immediately after observation using WLI and CE. We used the VS classification for the diagnosis of the HM with MENBI. According to this classification, we recognized the HM based on irregularity of the microsurface pattern or microvascular pattern in the lesion. Biopsies were taken from several points that appeared negative for cancer to ensure the HM diagnosis in all lesions in patients in the NBI group, and all biopsy specimens were confirmed as negative.

\section{Extraction of sections and pathological investigation}

We investigated the histological features of two slices in each case. The slices were selected as follows. For the ESD cases in the NBI group, we selected a representative point of the HM and took many endoscopic photographs with WLI, CE, and ME-NBI during the endoscopic observation period before ESD. We marked an incision using electrocautery devices on the point of the HM in the tumor to take representative pathological sections. For surgical cases, we 
extracted representative sections by comparing the endoscopic photographs and the postoperative formalin-fixed specimen after gastrectomy. All specimens were "pinned out" onto polystyrene receivers to facilitate subsequent histopathological sectioning before immediate fixation in $10 \%$ buffered formalin solution and cut into 2-mm-thick slices for ESD cases and 5-mm-thick slices for surgical cases. The fragments or slices were embedded in paraffin, cut into 3- $\mu \mathrm{m}$ sections, stained with hematoxylin and eosin (H\&E), and microscopically examined for histological type by expert pathologists (Y.I. and Y.T.). For the WLI/CE group, we selected the section of ESD specimen that was consistent with a landmark, such as the noticeable protuberance of type 0-IIa ECGs $(n=15)$ or clear indentation of type 0 -IIc $(n=13)$ in an endoscopic photograph.

We measured both the number and width of maximum distance between crypt openings, defined as the intervening part (IP), $2 \mathrm{~mm}$ from the borderline of the cancerous and noncancerous areas, at $40 \times$ magnification on the H\&Estained specimens. Additionally, to evaluate the capillary vessels, we performed CD34 immunostaining to measure the inner diameter of the minor axis to avoid bias from a diagonal cut, as well as the number of subepithelial capillaries (SECs) within $70 \mu \mathrm{m}$ from the surface, at $200 \times$ magnification, on CD34-stained specimens. We also measured the distance from the surface to the SEC.

\section{Statistical analysis}

Statistical analyses were performed using SPSS 24.0 (IBM, Armonk, NY, USA). Continuous variables were expressed as the mean \pm standard deviation (SD) or medians and ranges, and categorical parameters were expressed as numbers and frequencies. We used the Wilcoxon signedrank test to compare measured values of IP and SEC between cancerous and noncancerous areas. $P<0.05$ was considered statistically significant.

\section{Results}

A typical case from the NBI group is shown in Fig. 2. EGC (0-IIb type) was found in the gastric corpus, but the HM could not be clearly recognized with CE (Fig. 2a). By contrast, the ability of ME-NBI to visualize the irregularities of the microsurface and microvascular patterns enabled us to recognize the HM (Fig. 2b). For ESD, we marked dots around the lesion, and as indicated by the yellow arrow, marked a dot just outside the demarcation line according to the findings of ME-NBI. We then performed ESD (Fig. 2c). In the ESD specimen, the red lines indicate the extent of the cancerous tissue. The yellow arrow shows the marking dot on the demarcation line as above, indicating that the HM diagnosis was correct. Thus, we could compare the endoscopic images and pathological specimens using this dot as a marker (Fig. 2d).

Table 1 shows the clinicopathological characteristics of the patients and lesions. There were no significant differences in age, sex, tumor size, location, histological diagnosis, or depth of invasion between the NBI and WLI/CE groups.

Typical examples in which the number and width of the IP were measured with H\&E staining, the minor axis of SECs within $70 \mu \mathrm{m}$ from the surface was measured with CD34 immunostaining, and the distance from the surface to the SEC were measured are shown in Fig. 3a, b. The pathological findings of the surface crypt epithelial structure and surface vessels of the NBI group are shown in Table 2. There were no significant differences in mean IP width, SD, or number between cancer and noncancer areas [140.9:123.6 $\mu \mathrm{m}(p=0.170), 62.1: 53.3 \mu \mathrm{m}(p=0.212)$, and 13.0:14.2 $(p=0.366)$, respectively]. There were also no significant differences in the SEC minor axis or SD [6.9:7.1 $\mu \mathrm{m} \quad(p=0.813)$ and 2.4:2.4 $\mu \mathrm{m} \quad(p=0.978)$, respectively]. The number of SECs was significantly larger (33.2:23.8; $p=0.025)$, and the distance from the surface was significantly shorter $(26.4: 45.9 \mu \mathrm{m} ; p=0.001)$ in cancerous areas than in noncancerous areas.

A similar comparison of the WLI/CE group is shown in Tables 3 and 4. We subdivided this group into flat elevated-type (WLI/CE-IIa) and flat depressed-type (WLI/CEIIc) lesions. The IP width and SD were significantly larger, and the number was significantly smaller, in the cancerous area than in the noncancerous area in both the WLI/CE-IIa and WLI/CE-IIc groups. There were no significant differences in the SEC minor axis or SD between cancerous and noncancerous areas. SEC number was significantly larger in cancerous areas of the WLI/CE-IIc group, despite no significant difference being found in the WLI/CE-IIa group. The distance from the surface to the SEC was significantly shorter in cancerous areas than in noncancerous areas in both the WLI/CE-IIa and WLI/CE-IIc groups.

\section{Discussion}

In this study, we measured the number, width, and variation of the IP, and the number, width, variation, and depth of the SECs, in both cancerous and noncancerous areas on resected specimens of differentiated-type EGC that could only be detected with ME-NBI. We also investigated the same items for differentiated-type EGC that could be detected not only by ME-NBI but also by WLI and/or CE. Although we included several cases with a tumor that had invaded the submucosa, the invasion site was the center of the tumor, and the site of investigation was mucosal 
Fig. 2 Case with a 0-IIb lesion. a Chromoendoscopy findings of differentiated-type 0-IIb early gastric cancer. The horizontal margin could not be clearly recognized using white-light imaging and chromoendoscopy. b Findings of magnifying endoscopy with narrow-band imaging. By evaluating the irregularity of the microsurface and microvascular patterns, the horizontal margin can be clearly recognized (yellow dotted line). c We marked dots around the lesion; yellow arrow indicates a dot marked just outside the demarcation line according to the findings of magnifying endoscopy with narrow-band imaging. We then performed endoscopic submucosal dissection. d Endoscopic submucosal dissection specimen. Red lines show extent of cancerous tissue. Yellow arrow shows marking dot on demarcation line. The diagnosis of the horizontal margin was correct. We could compare the endoscopic images and pathological specimens using this dot as a landmark
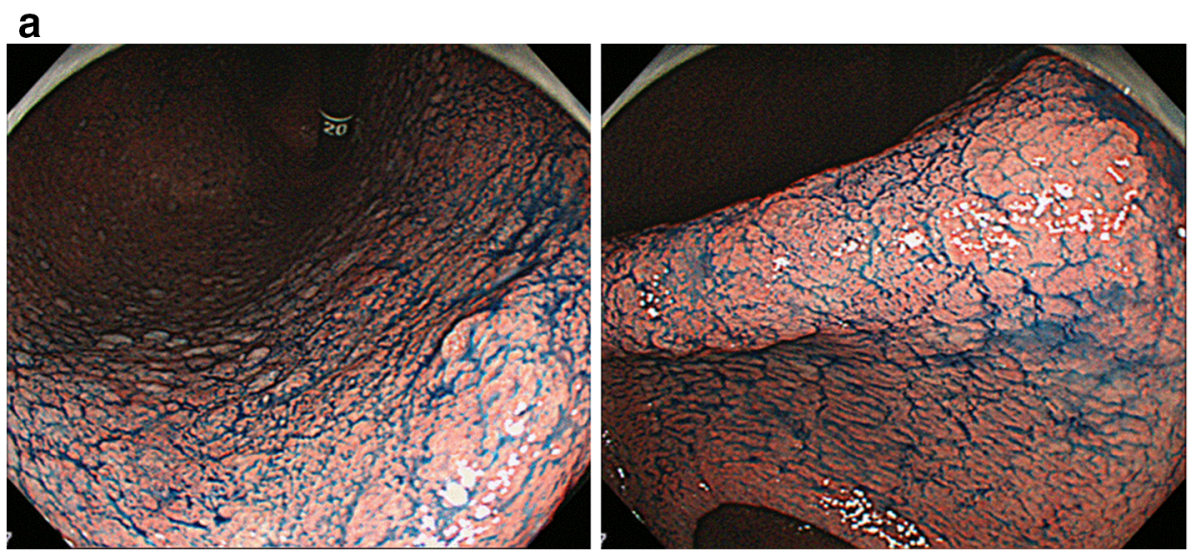

\section{b}
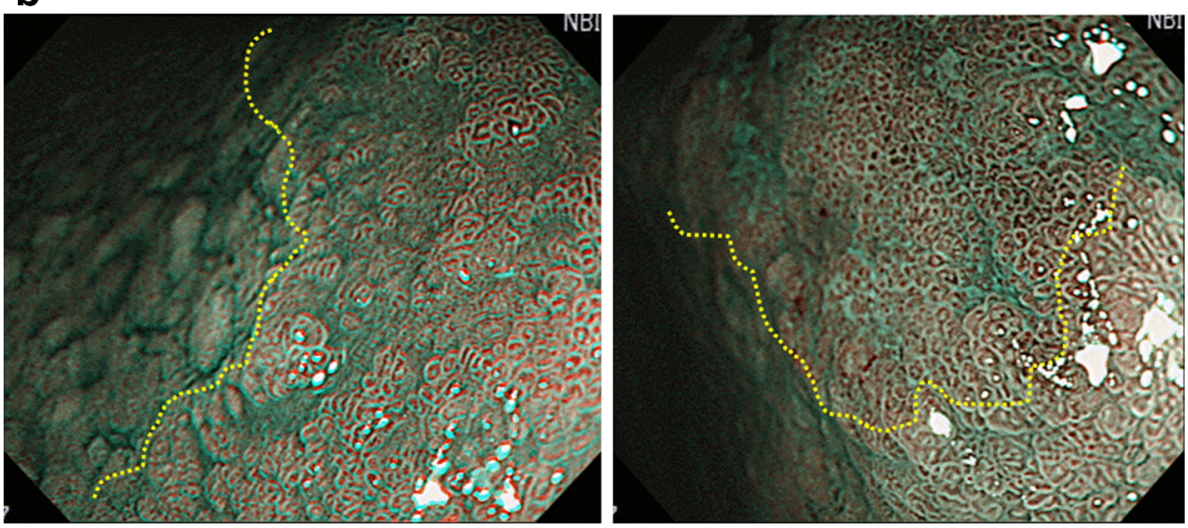

C
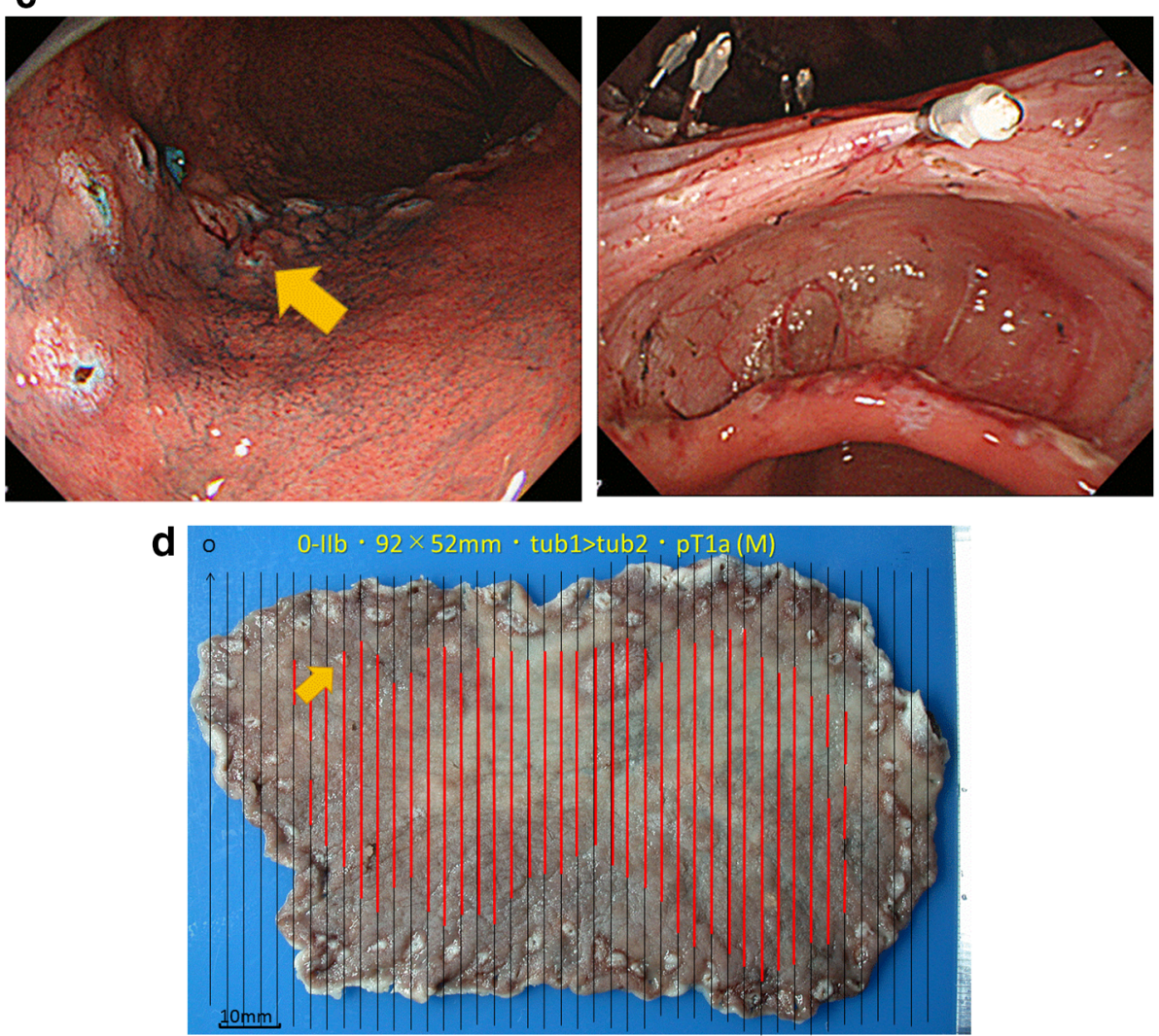
Table 1 Clinicopathological characteristics of the patients and lesions

\begin{tabular}{|c|c|c|c|}
\hline & NBI group $(n=11)$ & $\mathrm{WLI} / \mathrm{CE}$ group $(n=28)$ & $P$ value \\
\hline \multicolumn{4}{|l|}{ Patient characteristics } \\
\hline Mean age (range), years & $70.8(52-84)$ & $75.8(53-88)$ & $0.110^{*}$ \\
\hline Sex & & & $0.719 * *$ \\
\hline Male & 8 & 18 & \\
\hline Female & 3 & 10 & \\
\hline \multicolumn{4}{|l|}{ Lesion characteristics } \\
\hline Mean tumor size (range), $\mathrm{mm}$ & $57.7(25-95)$ & $50.5(35-98)$ & $0.405^{*}$ \\
\hline Location & & & $0.276^{* *}$ \\
\hline Upper & 1 & 7 & \\
\hline Middle & 8 & 10 & \\
\hline Lower & 2 & 11 & \\
\hline Histological diagnosis & & & - \\
\hline tub1, tub2, pap & 11 & 28 & \\
\hline sig, por & 0 & 0 & \\
\hline Depth of invasion & & & $0.648 * *$ \\
\hline Mucosa & 5 & 15 & \\
\hline Submucosa & 6 & 13 & \\
\hline
\end{tabular}

differentiated-type tubular adenocarcinoma. Therefore, the depth of invasion did not influence the identification of HM.

Our results suggested that in the WLI/CE group the IP was wide and scattered and the SEC extended to the surface in the cancerous area. These observations indicate that difference of IP width, SD, and IP number between cancerous and noncancerous lesions may be used to identify HMs with conventional endoscopy. Because NBI can allow better visualization of the vessel structure, the increased number of SECs and shallower SECs may allow clear observation of the HM with ME-NBI.

Several previous studies have reported the usefulness of ME-NBI for the precise diagnosis of superficial gastric cancers. For cases in which it was impossible to determine the presence and extent of cancer with conventional endoscopic examinations such as WLI and CE, ME-NBI may provide greater confidence about the accuracy, sensitivity, and specificity of the results [6-8]. The possibility of optical biopsy for small, depressed mucosal gastric cancer has also been suggested [7]. The principle of diagnosing superficial gastric cancer by ME-NBI is as follows: the lesion can be recognized with a demarcation line at first, and then the irregularity of the surface microvessels and micro-constructions can be observed in a demarcated area (VS classification) [6, 8]. The demarcation line has been similarly considered the same as that for the HM. Today, ME-NBI is coming to be widely used because of its usefulness for the recognition of the HM of the mucosal extent of EGC. The precise recognition of $\mathrm{HM}$ is essential to choosing an accurate treatment, including not only ESD but also surgery. The inaccurate diagnosis of HM might lead to incomplete resection and tumor recurrence in ESD cases and unnecessary total gastrectomy or cancer remnants in surgical cases. The standard conventional methods for determining HM are still WLI and CE [4, 6, 10]; however, these methods are not robust for recognizing the HM, especially in flat lesions such as type 0 -IIb. Although the frequency of predominant 0-IIb-type EGCs is rare $(0.4-8 \%)$ [3, 4], we often struggle to diagnose the margin for horizontal, extended lesions with flat components (IIb-like extended lesion) using conventional methods. The use of ME-NBI for IIb-like extended lesions has been reported to be more effective than conventional endoscopic imaging [4, 5, 10, 11].

Several studies have reported the usefulness and advantages of ME-NBI for diagnosing EGC using various expressions that describe the mucosal pattern or microvascular pattern [12-16]. Because these reports described only the classification of the appearance of the cancer pattern, for systematic diagnosis with ME-NBI, the VS classification is often used. When we use the VS classification in ME-NBI, we recognize the "difference" in the microvascular and microsurface patterns in the cancerous and noncancerous areas [8]. However, the determination of this "difference" depends on the 

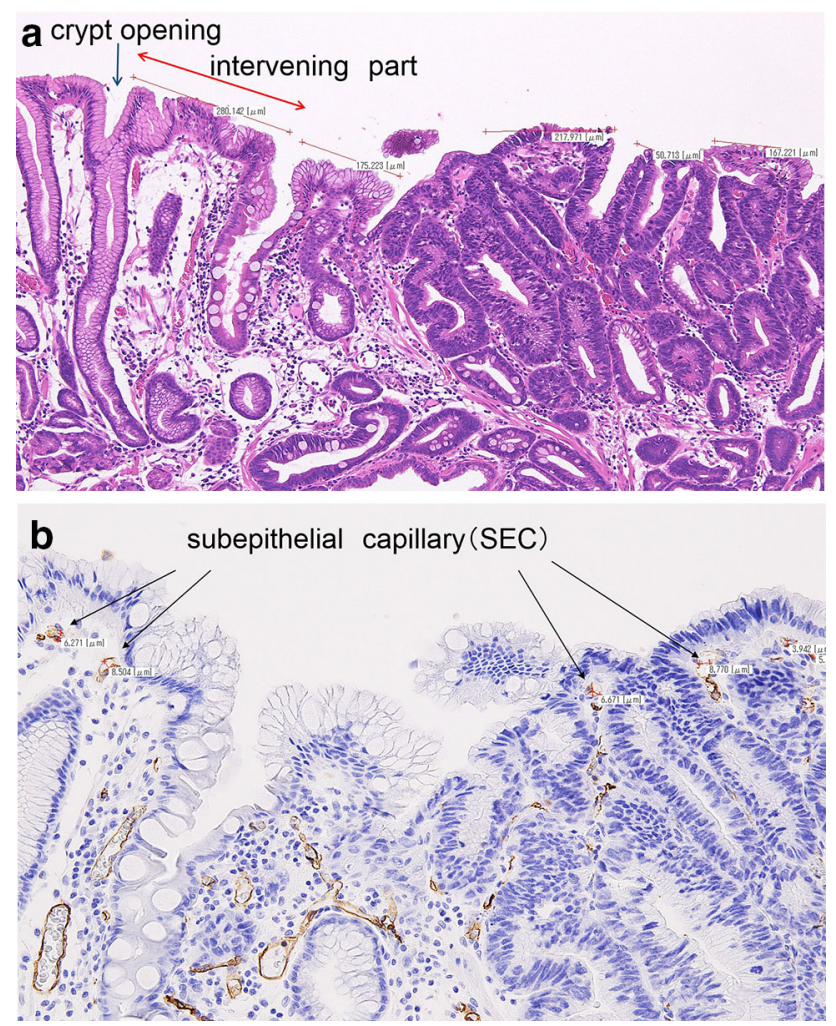

Fig. 3 Histological findings. a Measurement of the number and width of intervening parts at $40 \times$ magnification on hematoxylin and eosin-stained specimens. b Measurement of the minor axis, the number of subepithelial capillaries (SECs) within $70 \mu \mathrm{m}$ from the surface, and the distance from the surface to the SECs at $200 \times$ magnification on CD34-stained specimens

Table 2 Pathological findings in the NBI group

\begin{tabular}{lrcr}
\hline & Cancer & Noncancer & $P$ value \\
\hline IP width $(\mu \mathrm{m})$ & 139.8 & 123.9 & $0.110^{*}$ \\
IP width SD $(\mu \mathrm{m})$ & 48.0 & 44.2 & $0.355^{*}$ \\
IP number & 13.1 & 13.8 & $0.130^{*}$ \\
SEC minor axis $(\mu \mathrm{m})$ & 6.2 & 6.5 & $0.313^{*}$ \\
SEC minor axis SD $(\mu \mathrm{m})$ & 2.0 & 2.1 & $0.721^{*}$ \\
SEC number & 27.3 & 20.1 & $0.001^{*}$ \\
SEC depth $(\mu \mathrm{m})$ & 28.2 & 48.9 & $<0.001^{*}$
\end{tabular}

NBI narrow-band imaging, IP intervening part, SEC subepithelial capillaries

* Wilcoxon signed-rank test

subjective opinion of each endoscopist and the particulars of each case. Therefore, a study to define objective NBI findings is required, because there are few reports comparing ME-NBI and histological findings.

The present study identified the following two principal pathological findings that were consistent with ME-NBI findings. With regard to the IP, there were no significant differences in the width, number, and variation of the IP
Table 3 Pathological findings in the WLI/CE-IIa group

\begin{tabular}{lrcr}
\hline & Cancer & Noncancer & $P$ value \\
\hline IP width $(\mu \mathrm{m})$ & 250.5 & 122.5 & $<0.001^{*}$ \\
IP width SD $(\mu \mathrm{m})$ & 132.6 & 34.7 & $<0.001^{*}$ \\
IP number & 8.6 & 13.3 & $<0.001^{*}$ \\
SEC minor axis $(\mu \mathrm{m})$ & 6.3 & 7.0 & $0.126^{*}$ \\
SEC minor axis SD $(\mu \mathrm{m})$ & 2.3 & 2.3 & $0.681^{*}$ \\
SEC number & 19.6 & 18.7 & $0.489^{*}$ \\
SEC depth $(\mu \mathrm{m})$ & 33.8 & 46.2 & $0.002^{*}$
\end{tabular}

WLI/CE white-light imaging/chromoendoscopy, IP intervening part, $S E C$ subepithelial capillaries

* Wilcoxon signed-rank test

Table 4 Pathological findings in the WLI/CE-IIc group

\begin{tabular}{lrcr}
\hline & Cancer & Noncancer & $P$ value \\
\hline IP width $(\mu \mathrm{m})$ & 234.7 & 131.8 & $0.001^{*}$ \\
IP width SD $(\mu \mathrm{m})$ & 125.2 & 52.6 & $<0.001^{*}$ \\
IP number & 9.1 & 13.1 & $0.002^{*}$ \\
SEC minor axis $(\mu \mathrm{m})$ & 6.3 & 6.5 & $0.879^{*}$ \\
SEC minor axis SD $(\mu \mathrm{m})$ & 2.2 & 2.1 & $0.446^{*}$ \\
SEC number & 23.8 & 19.2 & $0.012^{*}$ \\
SEC depth $(\mu \mathrm{m})$ & 29.9 & 49.0 & $<0.001^{*}$
\end{tabular}

WLI/CE white-light imaging/chromoendoscopy, $I P$ intervening part, SEC subepithelial capillaries

* Wilcoxon signed-rank test

between cancerous and noncancerous tissue in the NBI group, although there were significant differences in the WLI/CE group. The distance from the mucosal surface to the SEC was significantly shorter in the cancerous area than in the noncancerous area, regardless of macroscopic type.

Insignificant differences in the IP width, SD, and number in the NBI group have led to confusion regarding HMs that are seen with conventional endoscopic images. On the other hand, the significant differences of IP width, SD, and number in the WLI/CE group were closely related to good recognition of the HM in clearly protuberant or depressed mucosal structures.

The present study also showed that SEC existed near the surface of the cancerous area in both groups, suggesting that in the cancerous area SEC can be seen more clearly than in the surrounding mucosa. The reason why SEC are present in the shallow layer is unknown. One hypothesis suggest that high expression of vascular endothelial growth factor (VEGF) may affect SEC growth. Although a few studies have investigated VEGF expression in advanced gastric cancers [17, 18], few reports have studied the 
expression of VEGF in EGC. A previous report that researched submucosal invasive gastric cancer showed that EGCs with an expansive, penetrating growth pattern tended to secrete VEGF, significantly inducing tumor angiogenesis; however, the VEGF-positive rate of superficially spreading gastric cancer growth was only 19.4\% [19]. Further investigation of the proliferation of blood vessels in early gastric cancer is needed.

We could not study microvascular findings in the present study, such as caliber variation, high density, disjoint direction, and abnormal branching, because we did not perform a three-dimensional analysis; however, because there was no significant difference regarding the caliber of the SEC within and outside the cancerous area in both groups, and the SECs were dense in the cancerous area in the NBI and WLI/CE-IIc groups, the irregular microvascular pattern may be atypical and caused by the proliferation of microvessels that extended close to the surface.

ME-NBI image processing has been reported [20]. This study was similar to the present study in that the microvessel density and mean diameter did not significantly differ between cancer and background tissue in an in vivo model.

Several other studies have investigated the condition of the microvessels in the cancerous and noncancerous areas using several image processing techniques. Two previous studies showed differences in blood flow between cancerous and noncancerous areas using a hemoglobin index to assess the histological type of gastric cancer [21, 22]. These reports revealed that the hemoglobin index ratio (cancerous area/noncancerous area) was higher in differentiated adenocarcinoma than in undifferentiated adenocarcinoma. These studies support the results of the present study, because SECs extended to the surface in the cancerous area and were denser, especially in the NBI group.

Adachi et al. [23] used a silicone rubber compound injection method with a methyl salicylate clearing technique in 32 resected early gastric carcinomas and observed the microvascular architecture under a stereomicroscope and light microscope. Compared to the surrounding normal mucosa, the differentiated carcinomas were mostly hypervascular or normovascular. The vascular size of the cancerous area was the same as that of the normal mucosa in approximately $60 \%$ of differentiated carcinomas, and vascular irregularity was absent in approximately $80 \%$. These findings also support those of the present study.

The limitations of the present study are as follows. First, the sample size was small. Second, the results were only applied to cancer in which the cancerous cells were exposed on the surface of the mucosa. In several types of EGCs, such as crawling cancer or undifferentiated-type cancer, cancer cells proliferate only in the middle layer of the mucosa, and abnormalities are not recognized at the surface; therefore, we cannot readily diagnose the HM in these types of cancer using NBI [4, 6], and we excluded patients with undifferentiated-type gastric cancer for these reasons.

In conclusion, in the present study, we showed that the distance from the surface to the SEC was significantly less in the cancerous area than in the noncancerous area, and the SEC were higher in density in the cancerous area than in the noncancerous area, especially in cases of 0 -IIb lesions. SEC can be seen more clearly in the cancerous area than in the noncancerous area, and ME-NBI may be useful to detect the HM by using these findings and lead to more accurate treatment, especially for 0-IIb lesions.

\section{Compliance with ethical standards}

Conflict of interest The authors declare that they have no conflict of interest.

\section{Guarantor of the article Kingo Hiraswa, $\mathrm{MD}, \mathrm{PhD}$}

Human rights statement and informed consent All procedures followed were in accordance with the ethical standards of the responsible committee on human experimentation (institutional and national) and with the Helsinki Declaration of 1964 and later versions. Informed consent or a substitute for it was obtained from all patients for being included in the study.

\section{References}

1. Gotoda T, Yamamoto H, Soetikno RM. Endoscopic submucosal dissection of early gastric cancer. J Gastroenterol. 2006;41:929-42.

2. Tajiri H, Niwa $H$. Proposal for a consensus terminology in endoscopy: how should different endoscopic imaging techniques be grouped and defined? Endoscopy. 2008;40:775-8.

3. Inoue $H$, Kashida $H$, Kudo S, Sasako M, Shimoda T, Watanabe H, Yoshida S, Guelrud M, Lightdale CJ, Wang K, Riddell RH, Diebold MD, Lambert R, Rey JF, Jung M, Neuhaus H, Axon AT, Genta RM, Gonvers JJ. The Paris endoscopic classification of superficial neoplastic lesions: esophagus, stomach, and colon: November 30 to December 1, 2002. Gastrointest Endosc. 2003;58:S3-43.

4. Nagahama T, Yao K, Maki S, Yasaka M, Takaki Y, Matsui T, et al. Usefulness of magnifying endoscopy with narrow-band imaging for determining the horizontal extent of early gastric cancer when there is an unclear margin by chromoendoscopy (with video). Gastrointest Endosc. 2011;74:1259-67.

5. Eleftheriadis $\mathrm{N}$, Inoue $\mathrm{H}$, Ikeda $\mathrm{H}$, Onimaru $\mathrm{M}$, Yoshida A, Maselli R, et al. Effective optical identification of type "0-IIb" early gastric cancer with narrow band imaging magnification endoscopy, successfully treated by endoscopic submucosal dissection. Ann Gastroenterol. 2015;28:72-80.

6. Yao K, Doyama H, Gotoda T, Ishikawa H, Nagahama T, Yokoi $\mathrm{C}$, et al. Diagnostic performance and limitations of magnifying narrow-band imaging in screening endoscopy of early gastric cancer: a prospective multicenter feasibility study. Gastric Cancer. 2014;17:669-79.

7. Ezoe Y, Muto M, Uedo N, Doyama H, Yao K, Oda I, et al. Magnifying narrowband imaging is more accurate than 
conventional white-light imaging in diagnosis of gastric mucosal cancer. Gastroenterology. 2011;141:2017-25.

8. Yao K, Anagnostopoulos GK, Ragunath K. Magnifying endoscopy for diagnosing and delineating early gastric cancer. Endoscopy. 2009;41:462-7.

9. Association Japanese Gastric Cancer. Japanese gastric cancer treatment guidelines 2010 (ver. 3). Gastric Cancer. 2011;14:113-23.

10. Kiyotoki S, Nishikawa J, Satake M, Fukagawa Y, Shirai Y, Hamabe $\mathrm{K}$, et al. Usefulness of magnifying endoscopy with narrow-band imaging for determining gastric tumor margin. J Gastroenterol Hepatol. 2010;25:1636-41.

11. Yao K, Nagahama T, Matsui T, Iwashita A. Detection and characterization of early gastric cancer for curative endoscopic submucosal dissection. Digest Endosc. 2013;25(Suppl 1):44-54.

12. Nonaka K, Namoto M, Kitada H, Shimizu M, Ochiai Y, Togawa $\mathrm{O}$, et al. Usefulness of the DL in ME with NBI for determining the expanded area of early-stage differentiated gastric carcinoma. World J Gastrointest Endosc. 2012;16:362-7.

13. Tatematsu H, Miyahara R, Shimoyama Y, Funasaka K, Ohno E, Nakamura $\mathrm{M}$, et al. Correlation between magnifying narrow-band imaging endoscopy results and organoid differentiation indicated by cancer cell differentiation and its distribution in depressed-type early gastric carcinoma. Asian Pac J Cancer Prev. 2013;14:2765-9.

14. Li HY, Dai J, Xue HB, Zhao YJ, Chen XY, Gao YJ, et al. Application of magnifying endoscopy with narrow-band imaging in diagnosing gastric lesions: a prospective study. Gastrointest Endosc. 2012;76:1124-32.

15. Nakayoshi T, Tajiri H, Matsuda K, Kaise M, Ikegami M, Sasaki H. Magnifying endoscopy combined with narrow band imaging system for early gastric cancer: correlation of vascular pattern with histopathology (including video). Endoscopy. 2004;36:1080-4.
16. Kanemitsu T, Yao K, Nagahama T, Fujiwara S, Takaki Y, Ono Y, et al. The vessels within epithelial circle (VEC) pattern as visualized by magnifying endoscopy with narrow-band imaging (ME$\mathrm{NBI}$ ) is a useful marker for the diagnosis of papillary adenocarcinoma: a case-controlled study. Gastric Cancer. 2014;17:469-77.

17. Tanigawa N, Amaya H, Matsumura M, Shimomatsuya T. Correlation between expression of vascular endothelial growth factor and tumor vascularity, and patient outcome in human gastric carcinoma. J Clin Oncol. 1997;15:826-32.

18. Maeda K, Kang SM, Onoda N, Ogawa M, Sawada T, Nakata B, et al. Expression of p53 and vascular endothelial growth factor associated with tumor angiogenesis and prognosis in gastric cancer. Oncology. 1998;55:594-9.

19. Tomoda M, Maehara Y, Kakeji Y, Ohno S, Ichiyoshi Y, Sugimachi K. Intratumoral neovascularization and growth pattern in early gastric carcinoma. Cancer (Phila). 1999;85:2340-6.

20. Araki Y, Sasaki Y, Hanabata N, Yoshimura T, Sawaya M, Hada $\mathrm{R}$, et al. Morphometry for microvessels in early gastric cancer by narrow band imaging-equipped magnifying endoscopy. Dig Endosc. 2011;23:233-9.

21. Yao K, Yao T, Matsui T, Iwashita A, Oishi T. Hemoglobin content in intramucosal gastric carcinoma as a marker of histologic differentiation: a clinical application of quantitative electronic endoscopy. Gastrointest Endosc. 2000;52:241-5.

22. Kim GH, Kim KB, Lim EK, Choi SH, Kim TO, Heo J, et al. Analysis of endoscopic electronic image of intramucosal gastric carcinoma using a software program for calculating hemoglobin index. J Korean Med Sci. 2006;21:1041-7.

23. Adachi Y, Mori M, Enjoji M, Sugimachi K. Microvascular architecture of early gastric carcinoma. Cancer (Phila). $1993 ; 72: 32-6$. 